\title{
Why Classical Schwarz Methods Applied to Hyperbolic Systems Converge even Without Overlap
}

\author{
V. Dolean ${ }^{1}$ and M.J. Gander ${ }^{2}$ \\ 1 Univ. de Nice Sophia-Antipolis, Laboratoire J.-A. Dieudonné, UMR CNRS No. \\ 6621, Nice, France. dolean@math .unice.fr \\ 2 Section de Mathématiques, Université de Genève, CP 240, 1211 Genève, \\ Martin.Gander@math.unige.ch
}

Summary. Overlap is essential for the classical Schwarz method to be convergent when solving elliptic problems. Over the last decade, it was however observed that when solving systems of hyperbolic partial differential equations, the classical Schwarz method can be convergent even without overlap. We show that the classical Schwarz method without overlap applied to the Cauchy-Riemann equations which represent the discretization in time of such a system, is equivalent to an optimized Schwarz method for a related elliptic problem, and thus must be convergent, since optimized Schwarz methods are well known to be convergent without overlap.

\section{Introduction}

The classical Schwarz method applied to scaler partial differential equations has been widely studied, as one can see from the many contributions in the proceedings of the international conferences on domain decomposition methods. Over the last decade, optimized variants of this method have been developed, which use absorbing conditions as transmission conditions at the interfaces between subdomains, and converge significantly faster than the classical Schwarz methods, see Gander [2006] and references therein. Less is known about the behavior of the classical Schwarz method applied to systems of partial differential equations; for the Euler equations, see Quarteroni [1990], Quarteroni and Stolcis [1996], Clerc [1998] and Dolean et al. [2002, 2004].

We show in this paper that the classical Schwarz method, which uses characteristic Dirichlet transmission conditions between subdomains, applied to the Cauchy Riemann equations is equivalent to an optimized Schwarz method applied to a well known equivalent elliptic problem. This explains why the classical Schwarz method in that case can be convergent even without overlap, and it allows us to develop more effective Schwarz methods for systems 
of partial differential equations. The extension of this idea to more realistic systems of PDEs, such as Maxwell equations both in the time-harmonic and time-discretized case, has already been done in Dolean et al. [2007].

\section{Cauchy-Riemann Equations and Scalar Equivalent}

To analyze the relationship between Schwarz methods for scalar partial differential equations (PDEs) and systems of PDEs, we use the Cauchy-Riemann equations

$$
\left\{\begin{array}{l}
\mathcal{L} \mathbf{u}:=\sqrt{\eta} \mathbf{u}+\left[\begin{array}{cc}
-1 & 0 \\
0 & 1
\end{array}\right] \partial_{x} \mathbf{u}+\left[\begin{array}{ll}
0 & 1 \\
1 & 0
\end{array}\right] \partial_{y} \mathbf{u}=\mathbf{f}, \text { on } \Omega=[0,1] \times \mathbb{R}, \\
v(0, y)=r(y), \quad u(1, y)=s(y), \quad y \in \mathbb{R} .
\end{array}\right.
$$

where $\mathbf{f}:=(f, g)^{T}, \mathbf{u}:=(u, v)^{T}$. The equations (1), even elliptic in nature, can be interpreted as the discretization in time of the following hyperbolic system:

$$
\partial_{t} \mathbf{u}+\left[\begin{array}{rr}
-1 & 0 \\
0 & 1
\end{array}\right] \partial_{x} \mathbf{u}+\left[\begin{array}{ll}
0 & 1 \\
1 & 0
\end{array}\right] \partial_{y} \mathbf{u}=0, \text { on } \Omega=[0,1] \times \mathbb{R} \times \mathbb{R}_{+} .
$$

One can imagine that at each time step, the resolution equations of type (1) is needed and therefore imposing the unknowns entering along the characteristics at the boundaries of the domain $\Omega$ like in (1) leads to a well-posed problem.

The following scalar boundary value problem

$$
\left\{\begin{array}{l}
\tilde{\mathcal{L}} \tilde{u} \equiv \eta \tilde{u}-\Delta \tilde{u}=\tilde{f}, \quad \text { in } \Omega, \\
\left(\partial_{x}-\sqrt{\eta}\right) \tilde{u}(0, y)=\tilde{r}(y), \quad \tilde{u}(1, y)=\tilde{s}(y), \quad y \in \mathbb{R}
\end{array}\right.
$$

is very much related to the Cauchy-Riemann equations:

Proposition 1. If $\tilde{f}=\left(\sqrt{\eta}+\partial_{x}\right) f-\partial_{y} g, \tilde{r}=\partial_{y} r-f(0, \cdot)$ and $\tilde{s}=s$, then the velocity component $u$ of the Cauchy-Riemann equations (1) coincides with the solution $\tilde{u}$ of the elliptic boundary value problem (2) for all $x, y \in \Omega$.

A similar elliptic PDE can also be derived for $v$, but we will not need it in the sequel.

\section{Classical Schwarz Algorithm}

We decompose the domain $\Omega$ into two overlapping or non-overlapping subdomains $\Omega_{1}=(0, b) \times \mathbb{R}$ and $\Omega_{2}=(a, 1) \times \mathbb{R}$, and we denote the overlap by $L:=b-a \geq 0$. A classical Schwarz algorithm for the Cauchy-Riemann equations (1) on these two subdomains is then defined by 


$$
\begin{aligned}
& \mathcal{L} \mathbf{u}_{1}^{n}=\mathbf{f}, \quad \text { in } \Omega_{1}, \quad \mathcal{L} \mathbf{u}_{2}^{n}=\mathbf{f}, \quad \text { in } \Omega_{2}, \\
& v_{1}^{n}(0, y)=r(y), \quad y \in \mathbb{R}, \quad u_{2}^{n}(1, y)=s(y), \quad y \in \mathbb{R}, \\
& u_{1}^{n}(b, y)=u_{2}^{n-1}(b, y), \quad y \in \mathbb{R}, \quad v_{2}^{n}(a, y)=v_{1}^{n-1}(a, y), \quad y \in \mathbb{R},
\end{aligned}
$$

where $\mathbf{u}_{j}^{n}=\left(u_{j}^{n}, v_{j}^{n}\right)$ denotes the n-th iterate of $\mathbf{u}$ in domain $\Omega_{j}, j=1,2$. Note that in this classical form of the Schwarz algorithm for the system of PDEs, we respected in the transmission conditions the information exchange along the characteristic directions, which is the most natural approach to follow when applying domain decomposition methods to hyperbolic problems, see for example Bjørhus [1995], Quarteroni and Stolcis [1996].

¿From the relation between the Cauchy-Riemann equations (1) and the associated elliptic problem (2) stated in Proposition 1, the related Schwarz algorithm for the elliptic problem is

$$
\begin{aligned}
& \tilde{\mathcal{L}} \tilde{u}_{1}^{n}=\tilde{f}, \quad \text { in } \Omega_{1} \quad \tilde{\mathcal{L}} \tilde{u}_{2}^{n}=\tilde{f}, \quad \text { in } \Omega_{2} \\
& \mathcal{B} \tilde{u}_{1}^{n}(0, y)=\tilde{r}(y), \quad y \in \mathbb{R}, \quad \tilde{u}_{2}^{n}(1, y)=\tilde{s}(y), \quad y \in \mathbb{R}, \\
& \tilde{u}_{1}^{n}(b, y)=\tilde{u}_{2}^{n-1}(b, y), \quad y \in \mathbb{R}, \quad \mathcal{B} \tilde{u}_{2}^{n}(a, y)=\mathcal{B} \tilde{u}_{1}^{n-1}(a, y), \quad y \in \mathbb{R}
\end{aligned}
$$

where $\mathcal{B}=\left(\partial_{x}-\sqrt{\eta}\right)$.

Theorem 1. If algorithm (4) is started with the initial guess $\tilde{u}_{1}^{0}=u_{1}^{0}$ and $\tilde{u}_{2}^{0}=u_{2}^{0}$, then the iterates of algorithm (4) and algorithm (3) coincide, $u_{l}^{n}(x, y)=\tilde{u}_{l}^{n}(x, y)$ for all $(x, y) \in \Omega_{l}, l=1,2$ and $n \geq 1$.

Proof. The proof is by induction. Proposition 1 entails the result for $n=1$. Assume then that the result is true at iteration $n-1$. Let $u^{1, n}, v^{1, n}, u^{2, n}$, and $v^{2, n}$ be the iterates of the Schwarz algorithm applied to the Cauchy-Riemann equations. We then have, on the one hand

$$
u^{1, n}(b, y)=u^{2, n-1}(b, y)=\tilde{u}^{2, n-1}(b, y)=\tilde{u}^{1, n}(b, y) .
$$

On the other hand, differentiating the interface condition on $v$ in (3) with respect to $y$ and using the first Cauchy-Riemann equation, we get

$$
\left(\partial_{x}-\sqrt{\eta}\right) u^{2, n}-f=\partial_{y} v^{2, n}=\partial_{y} u^{1, n-1}=\left(\partial_{x}-\sqrt{\eta}\right) u^{1, n-1}-f .
$$

When evaluating the above expression at $x=a$, the $f$ terms cancel, and we obtain

$$
\left(\partial_{x}-\sqrt{\eta}\right) u^{2, n}=\left(\partial_{x}-\sqrt{\eta}\right) u^{1, n-1}=\left(\partial_{x}-\sqrt{\eta}\right) \tilde{u}^{1, n-1}=\left(\partial_{x}-\sqrt{\eta}\right) \tilde{u}^{2, n} .
$$

Since the boundary conditions at $(0, y)$ and $(1, y)$ stay the same, the result follows from Proposition 1.

This theorem shows why the classical Schwarz algorithm (3) with characteristic Dirichlet transmission conditions for the Cauchy Riemann equations can converge even without overlap: it is equivalent to an optimized Schwarz 
method for a related elliptic PDE, and optimized Schwarz methods are also convergent without overlap, see Gander [2006].

We analyze now the convergence rate of Algorithm (3) when the domain is the entire plane, $\Omega=\mathbb{R}^{2}$, and the subdomains are $\Omega_{1}=(-\infty, L) \times \mathbb{R}$ and $\Omega_{2}=(0, \infty) \times \mathbb{R}, L \geq 0$. Let $\mathbf{e}_{l}^{n}(x, y)=\left(d_{l}^{n}(x, y), e_{l}^{n}(x, y)\right)^{t}:=\mathbf{u}(x, y)-$ $\mathbf{u}_{l}^{n}(x, y), l=1,2$ denote the error at iteration $n$. Then the $\mathbf{e}_{l}^{n}$ satisfy the homogeneous version of Algorithm (3), which after a Fourier transform $\mathcal{F}$ in $y$ with parameter $k, \hat{\mathbf{e}}_{l}^{n}:=\mathcal{F}\left(\mathbf{e}_{l}^{n}\right)$, gives

$$
\begin{aligned}
& \hat{\mathcal{L}} \hat{\mathbf{e}}_{1}^{n}=\mathbf{0}, \quad \text { in } \Omega_{1}, \quad \hat{\mathcal{L}} \hat{\mathbf{e}}_{2}^{n}=\mathbf{0}, \quad \text { in } \Omega_{2}, \\
& \hat{e}_{1}^{n}(-\infty, k)=0, \quad k \in \mathbb{R}, \quad \hat{d}_{2}^{n}(\infty, k)=0, \quad k \in \mathbb{R}, \\
& \hat{d}_{1}^{n}(L, k)=\hat{d}_{2}^{n-1}(L, k), k \in \mathbb{R}, \quad \hat{e}_{2}^{n}(0, k)=\hat{e}_{1}^{n-1}(0, k), k \in \mathbb{R},
\end{aligned}
$$

and $\hat{\mathcal{L}}$ denotes the action of the operator $\mathcal{L}$ after the Fourier transform in $y$, i.e.

$$
\hat{\mathcal{L}} \hat{\mathbf{u}}:=\mathcal{F}(\mathcal{L} \mathbf{u})=\sqrt{\eta} \hat{\mathbf{u}}+\left[\begin{array}{cc}
-1 & 0 \\
0 & 1
\end{array}\right] \partial_{x} \hat{\mathbf{u}}+\left[\begin{array}{ll}
0 & 1 \\
1 & 0
\end{array}\right] i k \hat{\mathbf{u}} .
$$

Theorem 2. If the initial error on the interfaces contains the Fourier components $\hat{\mathbf{e}}_{1}^{0}(L, k)$ and $\hat{\mathbf{e}}_{2}^{0}(0, k), k \in \mathbb{R}$, then for any overlap $L \geq 0$, algorithm (3) converges for all $k$,

$$
\left|\hat{\mathbf{e}}_{1}^{2 n}(L, k)\right|+\left|\hat{\mathbf{e}}_{2}^{2 n}(0, k)\right| \leq(\rho(\eta, L, k))^{2}\left(\left|\hat{\mathbf{e}}_{1}^{0}(L, k)\right|+\left|\hat{\mathbf{e}}_{2}^{0}(0, k)\right|\right),
$$

and the convergence factor is given by

$$
\rho(\eta, L, k)=\sqrt{\frac{\sqrt{\eta+k^{2}}-\sqrt{\eta}}{\sqrt{\eta+k^{2}}+\sqrt{\eta}}} e^{-L \sqrt{\eta+k^{2}}}<1, \quad \forall k \in \mathbb{R} .
$$

Proof. Solving (5) at iteration $n+1$, we obtain

$\hat{\mathbf{e}}^{1, n+1}=\alpha^{n+1} e^{\lambda(x-L)}\left(\begin{array}{c}\sqrt{\eta+k^{2}}+\sqrt{\eta} \\ -i k\end{array}\right), \hat{\mathbf{e}}^{2, n+1}=\beta^{n+1} e^{-\lambda x}\left(\begin{array}{c}-i k \\ \sqrt{\eta+k^{2}}+\sqrt{\eta}\end{array}\right)$,

where $\lambda=\sqrt{\eta+k^{2}}$ and $\alpha^{n+1}$ and $\beta^{n+1}$ are determined by the interface conditions to be

$$
\alpha^{n+1}=\beta^{n} \frac{-i k}{\sqrt{\eta+k^{2}}+\sqrt{\eta}} e^{-\sqrt{\eta+k^{2}} L}, \quad \beta^{n+1}=\alpha^{n} \frac{-i k}{\sqrt{\eta+k^{2}}+\sqrt{\eta}} e^{-\sqrt{\eta+k^{2}} L} .
$$

Performing a double step, this leads to the square of the convergence factor

$$
\rho(\eta, L, k)^{2}:=\frac{\alpha^{n+1}}{\alpha^{n-1}}=\frac{\beta^{n+1}}{\beta^{n-1}}=-\frac{\sqrt{\eta+k^{2}}-\sqrt{\eta}}{\sqrt{\eta+k^{2}}+\sqrt{\eta}} e^{-2 L \sqrt{\eta+k^{2}}},
$$

which implies the result by induction on $n$. 


\section{Optimized Schwarz Algorithm}

Algorithm (4) is a rather unusual optimized Schwarz algorithm for the elliptic problem (2), since it still uses Dirichlet transmission conditions at one of the interfaces. The guiding principle behind optimized Schwarz methods is to use absorbing transmission conditions, i.e. approximations of transparent boundary conditions at the interfaces between subdomains. The Robin transmission condition on one of the interfaces in (4) can be interpreted as a zeroth order low frequency approximation of a transparent condition, see Gander [2001]. In order to find better transmission conditions for the Cauchy-Riemann equations, we now derive their associated transparent boundary conditions.

To this end, we consider the Cauchy-Riemann equations (1) on the domain $\Omega=(0,1) \times \mathbb{R}$, with $\mathbf{f}=(f, g)^{T}$ compactly supported in $\Omega$, but with the new boundary conditions

$$
\left(v+\mathcal{S}_{1} u\right)(0, y)=0, \quad\left(u+\mathcal{S}_{2} v\right)(1, y)=0, \quad y \in R,
$$

where the operators $\mathcal{S}_{l}, l=1,2$ are general, pseudo-differential operators acting in the $y$ direction.

Lemma 1. If the operators $\mathcal{S}_{l}, l=1,2$, have the Fourier symbol

$$
\sigma_{l}:=\mathcal{F}\left(\mathcal{S}_{l}\right)=\frac{i k}{\sqrt{\eta}+\sqrt{\eta+k^{2}}}, \quad l=1,2
$$

then the solution of the Cauchy-Riemann equations (1) on the domain $\Omega=$ $(0,1) \times \mathbb{R}$ with boundary conditions (9) coincides with the restriction to the domain $\Omega$ of the solution of the Cauchy-Riemann equations (1) posed on $\mathbb{R}^{2}$.

Proof. It suffices to show that the difference between the solution of the global problem and the solution of the restricted problem vanishes. This difference, denoted by e, satisfies the homogeneous counterpart of (1) with boundary conditions (9), and its Fourier transform is

$$
\hat{\mathbf{e}}(x, k)=\alpha e^{\sqrt{\eta+k^{2}} x}\left(\begin{array}{c}
\sqrt{\eta+k^{2}}+\sqrt{\eta} \\
-i k
\end{array}\right)+\beta e^{-\sqrt{\eta+k^{2}} x}\left(\begin{array}{c}
-i k \\
\sqrt{\eta+k^{2}}+\sqrt{\eta}
\end{array}\right) .
$$

Now the first boundary condition in (9) implies $\beta \sqrt{\eta+k^{2}}=0$, and hence $\beta=0$, and the second one implies $\alpha \sqrt{\eta+k^{2}} e^{-\sqrt{\eta+k^{2}}}=0$, which gives $\alpha=0$, and hence $\hat{\mathbf{e}} \equiv 0$.

Remark 1. The symbols (10) can be written in several mathematically equivalent forms,

$$
\sigma_{l}=\frac{i k}{\sqrt{\eta}+\sqrt{\eta+k^{2}}}=\frac{\sqrt{\eta}-\sqrt{\eta+k^{2}}}{i k}=\sqrt{\frac{\sqrt{\eta}-\sqrt{\eta+k^{2}}}{\sqrt{\eta}+\sqrt{\eta+k^{2}}}} .
$$


The first form contains a local and a non-local term in $k$, since multiplication with $i k$ corresponds to derivation in $y$, which is a local operation (as the application of any polynomial in $i k$ would be), whereas the term containing the square-root of $k^{2}$ is a non-local operation. The second form contains two non-local operations, since the division by $i k$ corresponds to an integration. This integration can however be passed to the other variable in (9) by multiplication with $i k$. The last form contains only non-local terms. These different forms motivate different local approximations of the transparent boundary conditions, and thus lead to different optimized Schwarz methods, as we will show in the sequel.

We now consider the associated elliptic equations (2) on the domain $\Omega=$ $(0,1) \times \mathbb{R}$, with $f$ compactly supported in $\Omega$, but with the new boundary conditions

$$
\left(\partial_{x}-\tilde{\mathcal{S}}_{1}\right) u(0, y)=0, \quad\left(\partial_{x}+\tilde{\mathcal{S}}_{2}\right) u(1, y)=0, \quad y \in R,
$$

where the operators $\tilde{\mathcal{S}}_{l}, l=1,2$ are general, pseudo-differential operators acting in the $y$ direction.

Lemma 2. If the operators $\tilde{\mathcal{S}}_{l}, l=1,2$, have the Fourier symbol

$$
\tilde{\sigma}_{l}:=\mathcal{F}\left(\tilde{\mathcal{S}}_{l}\right)=\sqrt{\eta+k^{2}}, \quad l=1,2,
$$

then the solution of (2) on the domain $\Omega=(0,1) \times \mathbb{R}$ with boundary conditions (13) coincides with the restriction to $\Omega$ of the solution of (2) on $\mathbb{R}^{2}$.

Proof. The proof follows as in Lemma 1 using Fourier analysis.

Proposition 2. The velocity component $u$ of the solution of the CauchyRiemann equations (1) with boundary conditions (9), (10) coincides with the solution $\tilde{u}$ of the elliptic problem (2) with boundary conditions (13), (14) for all $x, y \in \Omega=(0,1) \times \mathbb{R}$.

Proof. We have already seen in Proposition 1 that the equations inside the domain coincide. It therefore suffices to show that the boundary conditions are also equivalent. By using the first Fourier transformed equation inside the domain, i.e. $i k \hat{v}=\left(\partial_{x}-\sqrt{\eta}\right) \hat{u}$, the boundary condition at $x=1$, i.e. $\left(\sqrt{\eta}+\sqrt{\eta+k^{2}}\right) \hat{u}+i k \hat{v}=0$, becomes $\left(\partial_{x}+\sqrt{\eta+k^{2}}\right) \hat{u}=0$, which is the transparent boundary condition for the elliptic equation. The same argument applies to the other boundary condition: using the first Fourier transformed equation, the boundary condition at $x=0$ becomes $\left(\sqrt{\eta}+\sqrt{\eta+k^{2}}\right)\left(\partial_{x}-\right.$ $\sqrt{\eta}) \hat{u}-k^{2} \hat{u}=0$. Taking into account that $k^{2}=\left(\sqrt{\eta+k^{2}}+\sqrt{\eta}\right)\left(\sqrt{\eta+k^{2}}-\right.$ $\sqrt{\eta})$, we further obtain $\left(\sqrt{\eta+k^{2}}+\sqrt{\eta}\right)\left(\sqrt{\eta+k^{2}}-\partial_{x}\right) \hat{u}=0$, which is equivalent to the transparent boundary condition for the scalar equation at $x=0$. 
We generalize now the classical Schwarz algorithm (3) by changing the transmission conditions at the interfaces,

$$
\begin{aligned}
\mathcal{L} \mathbf{u}_{1}^{n} & =0, \\
u_{1}^{n}(L, y)+\mathcal{S}_{1} v_{1}^{n}(L, y) & =u_{2}^{n-1}(L, y)+\mathcal{S}_{1} v_{2}^{n-1}(L, y), \\
\mathcal{L} \mathbf{u}_{2}^{n} & =0, \\
v_{2}^{n}(0, y)+\mathcal{S}_{2} u_{2}^{n}(0, y) & =v_{1}^{n-1}(0, y)+\mathcal{S}_{2} u_{1}^{n-1}(0, y) .
\end{aligned}
$$

Proceding as in Theorem 2, the convergence factor for a double iteration is

$$
\rho_{\text {opt }}\left(\eta, L, k, \sigma_{1}, \sigma_{2}\right)=\left|\frac{-i k+\sigma_{1}\left(\sqrt{\eta+k^{2}}+\sqrt{\eta}\right)}{\sqrt{\eta+k^{2}}+\sqrt{\eta}-i k \sigma_{1}} \frac{-i k+\sigma_{2}\left(\sqrt{\eta+k^{2}}+\sqrt{\eta}\right)}{\sqrt{\eta+k^{2}}+\sqrt{\eta}-i k \sigma_{2}} e^{-2 \sqrt{\eta+k^{2}} L}\right|^{\frac{1}{2}} .
$$

A good choice of $\sigma_{l}, l=1,2$ is a choice that makes the convergence factor $\rho_{\text {opt }}$ small for all values of $k$, and from (16), we see that the choice (10) is optimal, since then $\rho_{\text {opt }} \equiv 0$ for all $k$. But a good choice should also lead to transmission conditions which are as easy and inexpensive to use as the classical characteristic Dirichlet conditions. Guided by the equivalence with the scalar case, we will compare the following cases:

Case 1: $\sigma_{1}=\sigma_{2}=0$, the classical algorithm (3) with convergence factor (7).

Case 2: $\sigma_{1}=\frac{i k}{\sqrt{\eta}+p}, \sigma_{2}=\frac{\sqrt{\eta}-p}{i k}, p>0$, a mixed case, where the first form of the exact symbol in (12) is used to approximate $\sigma_{1}$ and the second form is used to approximate $\sigma_{2}$. This corresponds to first order transmission conditions, since $i k$ corresponds to a derivative in $y$ and the division by $i k$ can be avoided by multiplying the entire transmission condition by $i k$. The convergence factor is

$$
\rho_{2}(\eta, L, k, p)=\left|\left(\frac{\sqrt{\eta+k^{2}}-p}{\sqrt{\eta+k^{2}}+p}\right)^{2} e^{-2 \sqrt{\eta+k^{2}} L}\right|^{\frac{1}{2}},
$$

which is equivalent to the algorithm in the elliptic case with Robin transmission conditions $\partial_{x} \pm p$, see Gander [2001].

Case 3: $\sigma_{1}=\sigma_{2}=\sigma=\frac{i k}{\sqrt{\eta}+p}, p>0$, where only the first form of the exact symbol (12) has been used to approximate both $\sigma_{1}$ and $\sigma_{2}$. The resulting convergence factor is

$$
\rho_{3}(\eta, L, k, p)=\left|\frac{\sqrt{\eta+k^{2}}-\sqrt{\eta}}{\sqrt{\eta+k^{2}}+\sqrt{\eta}}\right|^{\frac{1}{2}} \rho_{2}(\eta, L, k, p)<\rho_{2}(\eta, L, k, p),
$$

and thus the convergence factor is smaller than in Case 2 by the same factor that was gained in Case 1 over the classical elliptic case.

Choosing the second form of the symbol (12) to approximate both $\sigma_{1}$ and $\sigma_{2}$ is not a good idea, since it inverts the additional low frequency factor, which is less than one in (18). 
Case 4: $\sigma_{1}=\frac{i k}{\sqrt{\eta}+p_{1}}, \sigma_{2}=\frac{\sqrt{\eta}-p_{2}}{i k}, p_{1,2}>0$, a choice motivated by Remark 1 , which leads to the convergence factor

$$
\rho_{4}\left(\eta, L, k, p_{1}, p_{2}\right)=\left|\frac{\sqrt{\eta+k^{2}}-p_{1}}{\sqrt{\eta+k^{2}}+p_{1}} \cdot \frac{\sqrt{\eta+k^{2}}-p_{2}}{\sqrt{\eta+k^{2}}+p_{2}} e^{-2 \sqrt{\eta+k^{2}} L}\right|^{\frac{1}{2}} .
$$

This corresponds to the two-sided Robin transmission conditions in the elliptic case in Gander [2001], which are of the form $\partial_{x}-p_{1}$ for the first subdomain and $\partial_{x}+p_{2}$ for the second one.

Case 5: $\sigma_{1}=\frac{i k}{\sqrt{\eta}+p_{1}}, \sigma_{2}=\frac{i k}{\sqrt{\eta}+p_{2}}, p_{1,2}>0$, which gives the even better convergence factor

$$
\rho_{5}\left(\eta, L, k, p_{1}, p_{2}\right)=\left|\frac{\sqrt{\eta+k^{2}}-\sqrt{\eta}}{\sqrt{\eta+k^{2}}+\sqrt{\eta}}\right|^{\frac{1}{2}} \rho_{4}\left(\eta, L, k, p_{1}, p_{2}\right)<\rho_{4}\left(\eta, L, k, p_{1}, p_{2}\right) .
$$

In the cases with parameters, the best choice for the parameters is in general the one that minimizes the convergence factor for all $k \in K$, where $K$ denotes the set of relevant numerical frequencies, for example $K=\left[k_{\min }, k_{\max }\right]$. One therefore needs to solve the min-max problems

$$
\min _{p>0} \max _{k \in K} \rho_{j}(\eta, L, k, p), j=2,3, \min _{p_{1}, p_{2}>0} \max _{k \in K} \rho_{j}\left(\eta, L, k, p_{1}, p_{2}\right), j=4,5 .
$$

In Case 2 and 4, the solution of the problem is already given in Gander [2001] for the equivalent elliptic case, and can therefore directly be used for the Cauchy-Riemann equations. The other cases are specific to the CauchyRiemann equations and an asymptotic analysis similar to the one shown in Gander [2001] leads to the results given in Table 1, where the estimate $k_{\max }=$ $\frac{C}{h}, C$ a positive constant, was used (a reasonable value would be $C=\pi$ ).

\begin{tabular}{|c|c|c|c|c|}
\hline & \multicolumn{2}{|c|}{ with overlap, $L>0$} & \multicolumn{2}{c|}{ without overlap, $L=0$} \\
\hline Case & $\rho$ & parameters & $\rho$ & parameters \\
\hline 1 & $1-2 \eta^{\frac{1}{4}} \sqrt{L}$ & none & $1-\frac{\sqrt{\eta}}{C} h$ & none \\
2 & $1-2^{\frac{13}{6}} \eta^{\frac{1}{6}} L^{\frac{1}{3}}$ & $p=\frac{2^{-\frac{1}{3}} \eta^{\frac{1}{3}}}{L^{\frac{1}{3}}}$ & $1-\frac{4 \eta^{\frac{1}{4}} \sqrt{h}}{\sqrt{C}}$ & $p=\frac{\sqrt{C} \eta^{\frac{1}{4}}}{\sqrt{h}}$ \\
3 & $1-2^{\frac{3}{2}} \eta^{\frac{1}{8}} L^{\frac{1}{4}}$ & $p=\frac{\eta^{\frac{4}{4}}}{\sqrt{L}}$ & $1-\frac{2^{\frac{4}{3}} \eta^{\frac{1}{6}}}{C^{\frac{1}{3}}} h^{\frac{1}{3}}$ & $p=\frac{2^{\frac{1}{3}} C^{\frac{2}{3}} \eta^{\frac{1}{6}}}{h^{\frac{2}{3}}}$ \\
4 & $1-2^{\frac{4}{5}} \eta^{\frac{1}{10}} L^{\frac{1}{5}}$ & $p_{1}=\frac{\eta^{\frac{1}{5}}}{2^{\frac{2}{5}} L^{\frac{3}{5}}}, p_{2}=\frac{\eta^{\frac{2}{5}}}{16^{\frac{1}{5}} L^{\frac{1}{5}}}$ & $1-\frac{\sqrt{2}^{\frac{1}{8}}}{C^{\frac{1}{4}}} h^{\frac{1}{4}}$ & $p_{1}=\frac{\sqrt{2} C^{\frac{3}{4}} \eta^{\frac{1}{8}}}{h^{\frac{3}{4}}}, p_{2}=\frac{C^{\frac{1}{4}} \eta^{\frac{3}{8}}}{\sqrt{2} h^{\frac{1}{4}}}$ \\
5 & $1-2 \eta^{\frac{1}{12}} L^{\frac{1}{6}}$ & $p_{1}=\frac{\eta^{\frac{1}{3}}}{L^{\frac{1}{3}}}, p_{2}=\frac{\eta^{\frac{1}{6}}}{L^{\frac{2}{3}}}$ & $1-\frac{2^{\frac{4}{5}} \eta^{\frac{1}{10}}}{C^{\frac{1}{5}}} h^{\frac{1}{5}}$ & $p_{1}=\frac{(2 C)^{\frac{4}{5}} \eta^{\frac{1}{10}}}{h^{\frac{4}{5}}}, p_{2}=\frac{(2 C)^{\frac{2}{5}} \eta^{\frac{3}{10}}}{h^{\frac{2}{5}}}$ \\
\hline
\end{tabular}

Table 1. Asymptotic convergence rate and optimal choice of the parameters in the transmission conditions for the five variants of the optimized Schwarz method applied to the Cauchy-Riemann equations, when the overlap $L$ or the mesh parameter $h$ is small, and the maximum numerical frequency is estimated by $k_{\max }=\frac{C}{h}$. 
One can clearly see in this table that there are much better transmission conditions than the characteristic ones for the Cauchy-Riemann equations: for a Schwarz algorithm with overlap of the order of the mesh parameter, $L=h$, the characteristic transmission conditions lead to a convergence factor $1-O(\sqrt{h})$, which depends strongly on $h$, whereas with better transmission conditions, one can achieve the convergence factor $1-O\left(h^{\frac{1}{6}}\right)$, which now depends only very weakly on $h$, at the same cost per iteration. Similar results also hold for the Schwarz algorithm without overlap, as shown in Table 1.

\section{Numerical Experiments}

We now show numerical experiments for the Cauchy-Riemann equations solved on the unit square $\Omega=(0,1) \times(0,1)$. We decompose the unit square into two subdomains $\Omega_{1}=(0, b) \times(0,1)$ and $\Omega_{2}=(a, 1) \times(0,1)$, where $0<a \leq b<1$, and therefore the overlap is $L=b-a$, and we consider both decompositions with and without overlap. We discretize the equations using the finite volume method on a uniform mesh with mesh parameter $h$. In all comparisons that follow, we simulate directly the error equations, $f=0$, and we use a random initial guess to ensure that all the frequency components are present in the iteration.

Table 2 shows the iteration count for all Schwarz algorithms considered, in the overlapping and non-overlapping case, and when the mesh is refined. These results are in good agreement with the theoretical results in Table 1:

\begin{tabular}{|c|c|c|c|c|c|c|c|c|}
\hline & \multicolumn{3}{|c|}{ with overlap, $L=3 h$} & \multicolumn{3}{|c|}{ without overlap, $L=0$} \\
\hline $\mathrm{h}$ & $1 / 32$ & $1 / 64$ & $1 / 128$ & $1 / 256$ & $1 / 32$ & $1 / 64$ & $1 / 128$ & $1 / 256$ \\
\hline Case 1 & 16 & 24 & 34 & 48 & 131 & 203 & 355 & 593 \\
Case 2 & 11 & 14 & 17 & 22 & 51 & 78 & 107 & 157 \\
Case 3 & 10 & 12 & 14 & 16 & 18 & 25 & 41 & 131 \\
Case 4 & 11 & 13 & 14 & 17 & 27 & 30 & 35 & 43 \\
Case 5 & 9 & 10 & 11 & 13 & 17 & 19 & 23 & 31 \\
\hline
\end{tabular}

Table 2. Number of iterations to attain convergence for different interface conditions and different mesh sizes in the overlapping and non-overlapping case. The tolerance is fixed at $\varepsilon=10^{-6}$.

the classical algorithm has the strongest dependence on the mesh parameter, and the other algorithms become less and less dependent.

\section{Conclusions}

We have shown for the Cauchy-Riemann equations that the classical Schwarz algorithm with characteristic Dirichlet transmission conditions can be con- 
vergent even without overlap. This is because it corresponds to a simple optimized Schwarz method for an equivalent elliptic problem, and optimized Schwarz methods are convergent without overlap. This relation reveals much more effective transmission conditions for hyperbolic problems and for their discretized in time counterparts (such as the Cauchy-Riemann equations) than the characteristic ones: convergence almost independent of the mesh parameter can be achieved with and without overlap. We are currently developing extensions of this approach to Maxwells equation.

\section{References}

Mørten Bjørhus. Semi-discrete subdomain iteration for hyperbolic systems. Technical Report 4, NTNU, 1995.

Sebastien Clerc. Non-overlapping Schwarz method for systems of first order equations. Cont. Math., 218:408-416, 1998.

Victorita Dolean, Luca Gerardo-Giorda, and Martin J. Gander. Optimized Schwarz methods for Maxwell equations, 2007. URL https://hal . archives-ouvertes.fr/hal-00107263. Submitted.

Victorita Dolean, Stephane Lanteri, and Frederic Nataf. Construction of interface conditions for solving compressible Euler equations by non-overlapping domain decomposition methods. Int. J. Numer. Meth. Fluids, 40:1485-1492, 2002.

Victorita Dolean, Stephane Lanteri, and Frederic Nataf. Convergence analysis of a Schwarz type domain decomposition method for the solution of the Euler equations. Appl. Num. Math., 49:153-186, 2004.

Martin J. Gander. Optimized Schwarz methods for Helmholtz problems. In Thirteenth international conference on domain decomposition, pages 245-252, 2001.

Martin J. Gander. Optimized Schwarz methods. SIAM J. Numer. Anal., 44(2): 699-731, 2006.

Alfio Quarteroni. Domain decomposition methods for systems of conservation laws: spectral collocation approximation. SIAM J. Sci. Stat. Comput., 11:1029-1052, 1990.

Alfio Quarteroni and L. Stolcis. Homogeneous and heterogeneous domain decomposition methods for compressible flow at high Reynolds numbers. Technical Report 33, CRS4, 1996. 